\title{
B-lymphocytes characterization in experimental autoimmune encephalomyelitis
}

\section{Fernanda Fossa (IC), Carolina Francelin (PQ), Alexandre Pereira (IC), Tábata Rodrigues (IC), Jéssica Funari (PG), Michelle Parise (PQ) e Alessandro Farias (PQ)}

\section{Abstract}

Multiple sclerosis (MS) is a chronic, inflammatory and demyelinating disease of the central nervous system (CNS), characterized as an autoimmune disease mediated by $\mathrm{CD}^{+} \mathrm{T}$ cells and studied with the aid of experimental autoimmune encephalomyelitis (EAE). Despite of the consensus that MS is evoked by an autoimmune reaction of $T$ lymphocytes, some new researches suggest that B-lymphocytes present a regulatory role at EAE, as well. Thus, in this study, we intended to characterize B-lymphocytes profile in the central nervous system (CNS) and lymph nodes during the clinical course of EAE.

Keywords: EAE, B lymphocytes, CNS

\section{Introduction}

Multiple sclerosis (MS) is a chronic, inflammatory and demyelinating disease of the central nervous system (CNS) against myelin protein and olygodendrocites. Clinically, it has periods of acute and remission, which causes inflammation and neurodegeneration (Stadelmann, 2011). The experimental autoimmune encephalomyelitis (EAE) is an experimental model usually used to study the clinical evolution of the disease in animals (Farias et al., 2013). B-lymphocytes are one of the cells involved in the disease development, which has shown contradictory roles in the pathogenesis of EAE and MS. Some studies show its pro inflammatory capacity (Hauser et al., 2008), while others point to an anti-inflammatory action of Blymphocytes (Matsushita et. al, 2008). Herein, our objective was to identify $B$ lymphocytes profile in the CNS during the clinical course of EAE.

\section{Results and Discussion}

Our flow cytometry analysis revealed the presence of B-lymphocyte $\left(\mathrm{OX}_{3}{ }^{+}\right)$in the CNS during the course of EAE. It was possible to observe that 7-10 days post immunization, Blymphocytes are predominantly in the lymph nodes while during the peak and remission phases there was an increase of $B$ cells in the CNS. In addiction, the gene expression study of $B$ cells [CD45R(B220 $)^{+}$CD19+] sorted from EAE's lymph nodes revealed the expression of IL-10 and TGF- $\beta$ cytokines. Until here, still remains unclear whether the regulatory function of B lymphocytes overlap or influence the immunopathogenesis of EAE.

\section{Conclusions}

We hypothesize that regulatory $B$ lymphocytes encountered in $E A E$ mice are involved with the remission phase process, helping to control the inflammation, which would lead to the remission of clinical signs. Thus, we need to investigate the exact mechanism, i.e., define if the role of these cells in EAE is pathogenic or protective and whether such cells are potential targets in the therapy of EAE.

\section{Acknowledgement}

We thank the Neuroimmunomodulation group, FAPESP (\#2011/18728-5), CNPQ and UNICAMP.

\footnotetext{
Stadelmann, C. Curr Opin Neurol, 2011. 24(3): p. 224-9.

${ }^{2}$ Farias A.S., Spagnol G.S., Bordeaux-Rego P., Oliveira C.O., Fontana AG, de Paula RF, Santos MP, Pradella F, Moraes AS, Oliveira EC, Longhini AL, Rezende AC,Vaisberg MW, Santos LM. CNS Neurosci. Ther. 2013 Apr;19(4):269-77.

${ }^{3}$ Hauser, S.L. and J.R. Oksenberg, Neuron, 2006. 52(1): p. 61-76.

${ }^{4}$ Matsushita, T., K. Yanaba, J.-D. Bouaziz, M. Fujimoto, and T. F. Tedder. 2008.. J. Clin. Invest. 118: 3420-3430.
} 